\title{
La démarche appréciative : une innovation pour dynamiser l'organisation
}

\author{
Pierre-Claude Élie, Consultant \\ Président Émergence Solutions
}

\section{INTRODUCTION}

La démarche appréciative s'est largement répandue dans le monde. Elle a été utilisée par des dizaines de milliers de personnes et des centaines d'organisations dans tous les secteurs de la société dans le but de promouvoir et d'implanter des changements et des transformations.

Depuis quelques années, la démarche appréciative s'est largement répandue dans le monde. Elle a été utilisée par des dizaines de milliers de personnes et des centaines d'organisations dans tous les secteurs de la société dans le but de promouvoir et d'implanter des changements et des transformations. Des entreprises de toutes tailles, des organismes gouvernementaux, des établissements de santé, des universités, des regroupements d'écoles, des organismes religieux et communautaires et de grandes organisations d'aide humanitaire ont utilisé la démarche avec succès.

Qu'est-ce qui peut bien inciter autant d'entreprises et d'organismes à utiliser la démarche appréciative quand ils font face à d'importants défis de changement? En se démarquant des méthodes traditionnelles centrées sur l'insatisfaction, les manques et les faiblesses et en se concentrant sur les forces vitales de l'organisation, la démarche appréciative dynamise et transforme les interventions déjà existantes en développement organisationnel comme la gestion du changement, la planification stratégique, la consolidation d'équipe, la restructuration, le design des processus d'affaires et bien d'autres. En faisant appel à l'intelligence et à la créativité collectives, la démarche appréciative accroît la capacité de l'organisation à changer, à instaurer un esprit de collaboration et à innover.

\section{En faisant appel à l'intelligence et à la créativité collectives, la démarche appréciative accroît la capacité de \\ l'organisation à changer, à instaurer un esprit de collaboration et à innover.}

\section{LA DÉMARCHE APPRÉCIATIVE : ACCROÎTRE LA CAPACITÉ À CHANGER, COLLABORER ET INNOVER DANS LES ORGANISATIONS}

La démarche appréciative est à la fois une approche pragmatique et un processus dynamique. Elle est une approche pragmatique qui se fonde sur des postulats articulés à partir de plusieurs recherches en psychologie et en management :

1. Chez tout individu et dans chaque organisation, quelque chose fonctionne bien. Ces facteurs de force peuvent être amplifiés et utilisés comme tremplins de changement et de croissance.

2. Dans nos conversations, nous construisons constamment notre réalité.
3. Nos questions et notre langage portent en eux les semences du changement.

4. Des images d'un futur attrayant engagent des actions positives.

5. Les émotions positives associées aux images du futur mobilisent l'énergie collective.

Quand on cherche autour de soi ce qu'il y a de meilleur chez les individus et dans les systèmes sociaux (équipes, groupes, organisations, communautés), les qualités ressortent, mais aussi ont tendance à se multiplier. Cette exploration incite à apprécier ce qui est enrichissant dans nos vies, 
dans nos relations et dans les situations que nous vivons.

La démarche appréciative propose un processus dynamique en quatre phases qui invite les gens à bâtir le type d'organisation et le monde dans lesquels ils veulent évoluer. Dans un esprit de coopération, le processus convie à découvrir ce qui dynamise une organisation quand elle est dans sa zone d'excellence, à explorer les rêves, les aspirations et le désir d'un avenir attrayant, et à utiliser ce nouveau savoir à des fins de mobilisation du personnel et d'un fonctionnement organisationnel optimal.

\section{LES ÉTAPES DE LA MISE EN EUVRE DE LA DÉMARCHE APPRÉCIATIVE}

\section{La démarche propose un processus en quatre phases (Découverte, Désir, Design et Destin) qui s'articulent autour d'un thème central exprimé en termes positifs. Le choix du thème est d'ailleurs crucial puisqu'il influence chacune des phases.}

La démarche propose un processus en quatre phases (Découverte, Désir, Design et Destin) qui s'articulent autour d'un thème central exprimé en termes positifs. Le choix du thème est d'ailleurs crucial puisqu'il influence chacune des phases. Le processus peut s'appliquer tout autant dans un entretien de coaching que dans un déroulement plus formel engageant l'ensemble des employés d'une organisation pendant plusieurs mois. Bien qu'il n'y ait pas de formule unique, le point de départ de toute démarche est un défi particulier de l'organisation dans un environnement qui lui est propre. Tel qu'illustré à la figure 1, la démarche appréciative, chaque étape vise des objectifs précis et mobilisateurs.

\section{Étape 1 : Découverte}

Au cours de cette étape, il s'agit de découvrir les forces et les facteurs de succès qui donnent à l'organisation toute sa vitalité lorsqu'elle excelle dans ce qu'elle fait. À l'aide d'entrevues, rechercher, explorer et apprendre des uns et des autres tout ce qui constitue le caractère unique de l'organisation et son essence vitale.

\section{Étape 2 : Désir}

À ce stade, il est conseillé d'imaginer et peindre le tableau d'un futur hautement désiré dans l'organisation. Énoncer en termes vivants de nouvelles possibilités qui inspireront des orientations décidées en fonction des aspirations, de l'environnement, des contributions et des images de la réussite.

Figure 1 : Étapes de mise en ouvre de la démarche appréciative

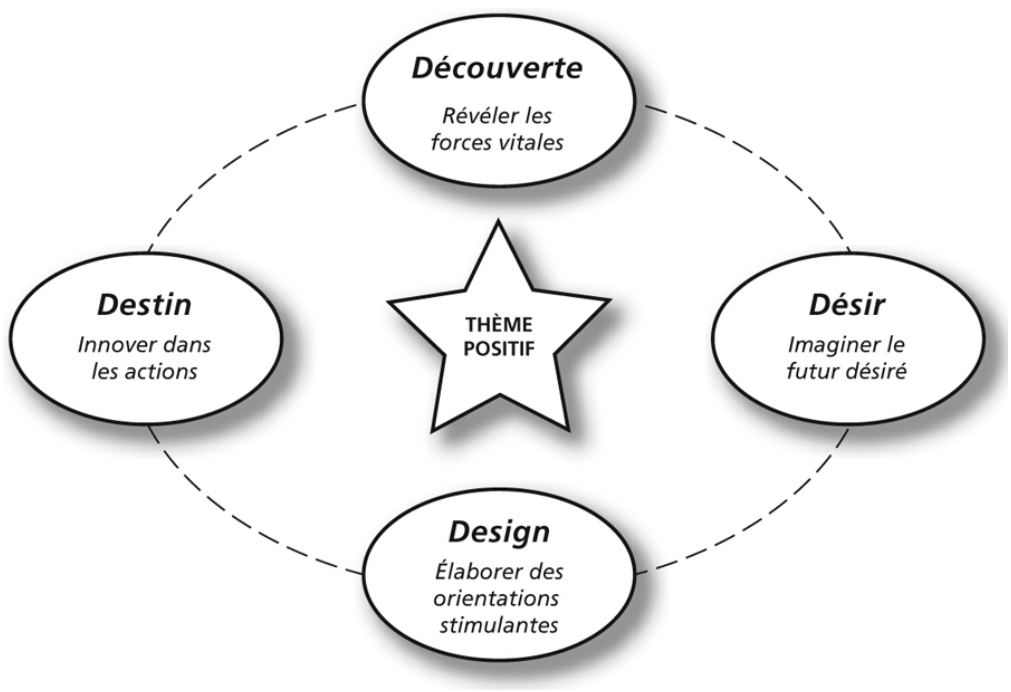




\section{Étape 3 : Design}

$\mathrm{Au}$ cours de cette étape, l'effort consiste à fonder les idéaux dans la réalité et bâtir la confiance nécessaire à leur atteinte. À cette fin, il est approprié d'utiliser et d'intégrer les découvertes, les images et les ressources individuelles et collectives pour déterminer ce que sera l'organisation. L'énoncé des propositions évocatrices et engageantes est nécessaire pour construire un projet futur prometteur et partagé.

\section{Étape 4 : Destin}

Enfin, il s'agit de mettre en place des actions innovatrices, des mécanismes, des programmes et des processus mobilisateurs pour tirer le meilleur parti des ressources et des talents de chacun. À cet égard, il est pertinent d'entretenir une culture mettant l'accent sur l'appréciation dans les interactions entre les gens et dans les façons de faire.

\section{POURQUOI LA DÉMARCHE APPRÉCIATIVE FONCTIONNE-T-ELLE?}

\section{La démarche génère plus d'innovations, une productivité améliorée, une plus grande satisfaction chez les employés, une coopération accrue entre des groupes de travail diversifiés, et souvent une meilleure profitabilité pour l'organisation.}

$\mathrm{Au}$ cours des dernières années, le travail de nombreux chercheurs et consultants qui ont utilisé la démarche appréciative a permis de constater des transformations très marquantes dans la façon qu'ont les gens d'interagir entre eux et dans les résultats qu'ils obtiennent. La démarche génère plus d'innovations, une productivité améliorée, une plus grande satisfaction chez les employés, une coopération accrue entre des groupes de travail diversifiés, et souvent une meilleure profitabilité pour l'organisation.

Plusieurs arguments sont à la base de cette affirmation. D'abord, explorer les aspects positifs d'une situation et dialoguer sur les forces et les possibilités est beaucoup plus engageant pour les participants que de parler des problèmes à régler. Ensuite, les histoires partagées avec d'autres créent des connexions uniques qui contribuent à révéler l'unicité des individus et les forces vitales de l'organisation. L'approche appréciative permet aussi de valoriser les collaborateurs. En effet, les participants se sentent écoutés et valorisés. Le sentiment de confiance en eux-mêmes est rehaussé. Aussi, les images positives qui émergent dans les conversations et les remue-méninges incitent à l'action et favorisent de nouveaux comportements. Par ailleurs, parce que le futur s'articule sur les

réussites du passé et du présent, l'organisation gagne en confiance et en inspiration et peut donc continuer à progresser vers l'avenir avec une conscience accrue des facteurs qui mènent à la réussite. Enfin, la vision nouvelle résultant de la démarche ainsi que les liens qui se sont établis génèrent souvent des sentiments de joie et de fierté qui ont un effet contagieux dans l'ensemble de l'organisation.

Plusieurs praticiens ont constaté que la démarche libérait de nouveaux pouvoirs souvent inconnus jusqu'alors tant par les individus que par l'organisation. En voici quelques-uns : le pouvoir d'être écouté; le pouvoir d'être reconnu dans son unicité; le pouvoir de rêver collectivement; le pouvoir de contribuer par ses idées et ses actions; le pouvoir d'agir en ayant du soutien; le pouvoir d'être positif.

\section{Plusieurs praticiens ont constaté que la démarche libérait de nouveaux pouvoirs souvent inconnus jusqu'alors tant par les individus que par l'organisation.}

La puissance de l'approche appréciative provient en grande partie de sa capacité à transformer les perceptions individuelles en une nouvelle réalité de groupe. Grâce à leurs conversations, les gens construisent ensemble le système social (équipe, groupe, organisation, communauté) dans lequel ils sont engagés. Ils influencent ainsi plusieurs processus, dont la communication organisationnelle, la gestion des conflits, la prise de décision, l'interaction entre les personnes et la gestion du changement. 


\section{LA DÉMARCHE APPRÉCIATIVE EN PRATIQUE}

\author{
Au Canada et au Québec, des organismes \\ gouvernementaux et paragouvernementaux, des \\ associations, des établissements de santé, des \\ commissions scolaires, des universités, des \\ organismes communautaires, de grandes et \\ petites entreprises ont utilisé avec succès la \\ démarche appréciative.
}

Des utilisations de la démarche appréciative ont été faites dans de nombreux domaines, tels que la productivité, l'innovation, la planification stratégique, les fusions, l'implantation de nouveaux systèmes, la restructuration, la culture organisationnelle, le service à la clientèle, la collaboration dans les équipes, la mobilisation $\mathrm{du}$ personnel, la réussite de projets et le développement des compétences.

L'approche s'est avéré un puissant moteur de changement positif pour un grand nombre d'entreprises et d'organismes humanitaires dans le monde. Au Canada et au Québec, des organismes gouvernementaux et paragouvernementaux, des associations, des établissements de santé, des commissions scolaires, des universités, des organismes communautaires, de grandes et petites entreprises ont utilisé avec succès la démarche appréciative. Certains l'ont même intégré à leur mode de gestion. Afin d'illustrer de façon concrète des résultats obtenus dans diverses organisations, voici quelques cas vécus au cours des dernières années.

Cas 1 : Association paritaire pour la santé et la sécurité du travail du secteur affaires sociales (ASSTSAS)

Défi : Comment inviter une équipe de cadres, de professionnels de la santé et d'employés (30 personnes) à devenir une équipe extraordinaire et reconnue pour la haute qualité de ses interventions et comme un phare dans le milieu? Actions : En collaboration avec une équipe de quatre cadres, un atelier d'une journée a été conçu pour sensibiliser les participants à la démarche appréciative, pour imaginer ce qu'ils souhaitaient devenir et décider d'orientations stratégiques pour concrétiser ce futur désiré. L'équipe de professionnels a participé acti- vement à l'élaboration de l'intervention dans les semaines précédant l'évènement. Tout le processus a été fait dans un grand esprit de collaboration créative. Cette approche a permis de transmettre de nombreuses connaissances à l'équipe. Trois d'entre eux sont allés se perfectionner aux États-Unis à la suite de l'intervention. Un an après la tenue de cet atelier, l'ASSTAS a publié un numéro spécial de sa revue Prévention entièrement consacré à des projets avec des clients au cours desquels des intervenants avaient utilisé la démarche appréciative.

L'équipe de professionnels a participé activement à l'élaboration de l'intervention dans les semaines précédant l'évènement. Tout le processus a été fait dans un grand esprit de collaboration créative.

\section{Cas 2: Centre universitaire de santé McGill (CUSM) (regroupement de cinq grands hôpitaux)}

Défi : Comment amener une équipe de cadres supérieurs hautement spécialisés en soins infirmiers et provenant de cinq hôpitaux différents à collaborer pour se donner une vision et un cadre de référence communs?

Actions : Intervention de planification stratégique avec le Comité de direction des Soins infirmiers du CUSM ayant pour objectifs de définir une vision partagée, de décider d'une nouvelle orientation commune à cinq hôpitaux dans un contexte en pleine transformation. Il s'agit aussi d'établir un nouveau modèle de soins unique pour les cinq établissements rattachés à McGill en accord avec la nouvelle vision centrée sur les patients et leur famille et les nouvelles exigences du Ministère de la Santé. L'intervention cherchait aussi à éliminer les obstacles générés par certains membres du comité, à développer un plan d'action réaliste et réalisable, à amener l'ensemble du comité à adhérer au plan et à s'engager à le mettre en œuvre. L'intervention a permis non seulement d'atteindre les objectifs fixés, mais aussi de souder une équipe formée de personnes venant de cultures et d'univers divers. 
Cas 3: Cirque du Soleil (entreprise de divertissement à l'échelle mondiale)

Défi : Comment concevoir un parcours d'apprentissage en leadership qui soit utilisable partout dans le monde dans un contexte de croissance rapide et de tensions importantes entre cadres et créateurs avec une entreprise n'ayant pas de culture de leadership et reconnue pour son originalité et sa grande créativité?

Actions : En collaboration avec le service des ressources humaines et du développement organisationnel, une intervention globale a été conçue afin de définir dans un premier temps l'essence du leadership dans l'organisation. Le but poursuivi étant de bâtir un programme de formation en leadership pour l'ensemble des cadres dans le monde. Des entrevues en mode appréciatif ont été réalisées avec douze membres de la haute direction et une centaine de cadres intermédiaires travaillant au siège social et à l'étranger. La démarche appréciative a permis de définir les six compétences clés en leadership qui avaient contribué au succès du Cirque et les six nouvelles compétences nécessaires pour mener le Cirque vers ses nouveaux objectifs de développement à l'international. Une deuxième phase d'intervention a permis d'imaginer et de concevoir un parcours d'apprentissage tout à fait unique adapté pour trois niveaux hiérarchiques de gestion. Le parcours a intégré les valeurs et les forces génératrices d'excellence dans l'organisation, ainsi que d'autres éléments typiques qui font partie de la mythologie du Cirque du Soleil.

\section{La démarche appréciative a permis de définir les six compétences clés en leadership qui avaient contribué au succès du Cirque et les six nouvelles compétences nécessaires pour mener le Cirque vers ses nouveaux objectifs de développement à l'international.}

Cas 4 : Hôpital de Montréal pour enfants (un des deux hôpitaux pour enfants à Montréal)

Défi : Comment amener toute l'équipe de gestion (72 cadres) du service des soins infirmiers d'un grand hôpital à attirer les meilleurs talents et à les retenir dans un contexte de pénurie d'effectifs?
Actions : En collaboration avec un comité spécialement constitué, nous avons conçu et animé un atelier de deux jours ayant pour objectifs d'amener les gens à identifier leurs forces vives lorsqu'ils sont dans leur zone d'excellence; à imaginer une vision commune; à décider d'orientations stratégiques; à rédiger des propositions stimulantes reliées à des domaines précis et à prendre des engagements dans la mise en œuvre d'actions concrètes.

En fin de parcours, des comités volontaires se sont formés pour mettre en place des actions en lien avec une proposition très claire: celle de devenir un hôpital magnétique (attrayant) et un des cinq meilleurs hôpitaux de la région de Montréal.

\section{Cas 5 : Ministère des Ressources naturelles et de la Faune (MRNF)}

Défi : Comment amener une nouvelle équipe de cadres supérieurs à travailler en cohésion dans un contexte de changement structurel?

Actions : Le MRNF venait de mettre en place une nouvelle structure administrative favorisant une plus grande autonomie des régions. De nouveaux directeurs généraux régionaux (DGR) furent alors engagés. Une de leurs responsabilités était de rassembler sous une même direction dans chacun des 13 territoires du Québec les cinq divisions du Ministère. Dans un premier temps, à l'aide d'entrevues, nous avons évalué le contexte organisationnel et la culture du Ministère tant au siège social que dans les régions. Dans un deuxième temps, nous avons tenu un Lac à l'épaule avec les nouveaux directeurs généraux dont l'objectif était de créer un haut degré de collaboration entre les directeurs venant d'horizons divers. L'utilisation de la démarche appréciative comme cadre d'intervention a permis aux participants de reconnaître leurs forces et leurs points de convergence, d'énoncer leurs aspirations et dessiner une vision commune quant à leur rôle, leurs responsabilités, les relations entre eux et avec le centre administratif à Québec, d'élaborer et proposer des orientations très stimulantes et de définir des mécanismes de fonctionnement et de collaboration entre eux dans l'avenir. 


\section{CONCLUSION}

\section{L'expérimentation de la démarche appréciative démontre qu'il est beaucoup plus difficile d'utiliser une telle approche dans un milieu hautement hiérarchisé, où le statut des gestionnaires est très important et où l'on pratique encore un style de gestion s'appuyant sur l'autorité et le contrôle.}

La démarche appréciative fonctionne bien si les dirigeants qui parrainent l'intervention manifestent une ouverture à des valeurs fondées sur le dialogue, l'écoute attentionnée, le partage des expériences positives, la créativité et l'imagination collectives, l'innovation et une saine dynamique entre les participants. Cependant, l'expérimentation de la démarche appréciative démontre qu'il est beaucoup plus difficile d'utiliser une telle approche dans un milieu hautement hiérarchisé, où le statut des gestionnaires est très important et où l'on pratique encore un style de gestion s'appuyant sur l'autorité et le contrôle. 